\title{
Brachio- Axillary Translocation Fistula with Reverse Saphenous Venous Graft: a New Hope for the Patients of End stage Renal Disease
}

\author{
SMG Saklayen Russel ${ }^{1}$, Jubayer Ahmad ${ }^{2}$, Raju Ahmed ${ }^{3}$, Jashim Uddin ${ }^{4}$, Suman Nazmul Hosain ${ }^{5}$
}

\begin{abstract}
:
Native arterio-venous fistula (AVF) are the preferred mode of repeated vascular access for the chronic renal failure patients surviving on hemodialysis because of their easy accessibility, good long term patency, low complication rate and cost-effectiveness. Creation of a fistula between the radial or brachial artery and a suitable adjacent vein is the most commonly practiced option. However the major upper arm veins of the CKD patients are often found thrombosed, cord like and not suitable for AV anastomosis. A 48 years old male patient of chronic kidney disease with a permanent catheter placed in the right subclavian vein was referred to create an AV fistula. On exploration none of the upper
\end{abstract}

limb veins was found suitable for fistula formation. The proximal part of the left GSV was harvested from patient's left upper thigh and was used to make a connection between left brachial artery at cubital fossa and the left axillary vein. To avoid over flooding of the limb vasculature, partial banding of the left axillary vein was done distal to this anastomosis. When all options in both upper limbs are exhausted, autologous great saphenous grafts may be a very useful tool for the surgeons in creating upper limb AV fistulas in difficult situations.

Keywords: Arteriovenous fistula, autogenous AVF, graft AVF, Great Saphenous Vein.
Introduction:

Repeated vascular access is an obligatory requirement for the chronic renal failure patients surviving on hemodialysis. Native arterio-venous fistula (AVF) are the preferred mode of vascular access for the maintenance of haemodialysis (HD) in the patients with end stage renal disease because of their easy accessibility, good long term patency and low complication rate $^{1}$. It is also cost effective for the patient. The high frequency

1. Assistant Professor and Associate Consultant, Dept. of Vascular Surgery, Ibrahim Cardiac Hospital and Research Institute, Dhaka.

2. Registrar, Dept. of Vascular Surgery, Ibrahim Cardiac Hospital and Research Institute, Dhaka.

3. Assistant Prof of Anaesthesia, Ibrahim Cardiac Hospital and Research Institute, Dhaka.

4. Registrar, Dept of Anaesthesia, Ibrahim Cardiac Hospital and Research Institute, Dhaka.

5. Head of the Department of Cardiac Surgery, Chittagong Medical College \& Hospital, Chittagong.

Corresponding Address: Dr SMG Saklayen Russel, Assistant Professor and Associate Consultant, Dept. of Vascular Surgery, Ibrahim Cardiac Hospital and Research Institute, Dhaka. Email: saklayendmc@gmail.com. of thrombosis and infection associated with prosthetic channels also make native fistula the first choice. As it is the best access for longevity and has the lowest association with morbidity and mortality, this is strongly recommended by guidelines from different countries ${ }^{2}$. Creation of a fistula between the radial artery and a suitable adjacent vein is the most commonly practiced option. Next choice is fistula involving more proximal vessels with larger caliber like brachio-basilic fistula. However the problem is the major upper arm veins of the Chronic Kidney Disease patients are often found thrombosed, cord like and not suitable for AV anastomosis. Alternate maneuvers involving more proximal veins may be engaged to overcome the situation. Their vascular access also needs maintenance and management of various related complications. There are a large number of patients in whom AVF has either failed in both upper limbs or is not feasible because of unsuitable veins. The management of these patients to get a good long-term vascular access has been a matter of debate and discussion over the years ${ }^{1}$. 


\section{Case report:}

A 48 years old male patient of chronic kidney disease, diabetes mellitus and hypertension with a permanent catheter placed in the right subclavian vein was referred to the department of Vascular Surgery of Ibrahim Cardiac Hospital and Research Institute to create an AV fistula for the maintainance of haemodialysis. Duplex study of right upper limb vessels couldn't reveal any flow in the cephalic vein either on arm or forearm. Only left basilic vein was visible at the arm level with a diameter of $3 \mathrm{~mm}$ on duplex evaluation. So we planned for left Brachio-Basilic transposition fistula. All base line investigations were done. $S$ Creatinin level was 9.3. Patient was found to be both HBsAg positive and Anti-HCV positive. He is an ex-drug abuser.

After informed consent the patient underwent surgery with aseptic precaution on $18^{\text {th }}$ July 2017 . Surgical team took extra-precaution due to the seropositive status of the patient with both hepatitis B and C virus. Initially a brachial block and local anesthesia with injection lignocaine was used to create a brachio-basilic transposition fistula according to original operative plan. But on exploration the left Basilic vein was also found to be cord like and not suitable for anastomosis contradicting the preoperative duplex findings. So the operative plan had to be changed.

The patient and the party were briefed about the situation. They were offered Brachio-Basilic fistula with a prosthetic interposition graft. But patient refused that option due to financial constraint and the possible complications related to prosthetic graft. So we offered them for Brachio-Basilic translocation fistula with autologous graft i.e Great Saphenous Vein (GSV). Patient party agreed to do it. For this additional procedure the brachial block and local anesthesia wouldn't suffice. So the anesthetists had applied general anesthesia with laryngeal mask. The proximal part of the left GSV was harvested from patient's left upper thigh. End to side anastomosis was first made with the distal end of the harvested saphenous vein segment with the brachial artery at cubital fossa. This segment of vein was then passed through a subcutaneous tunnel to the left axillary region. End to side anastomosis was made with the proximal end of the harvested segment of GSV with the left axillary vein. To avoid over flooding of the limb vasculature, partial banding of the left axilary vein was done distal to this anastomosis. After proper haemostasis wound closed in layers kept a drain tube in situ. Good bruit and thrill found. The next day the drain was removed and the patient was discharged in good shape.

The postoperative clinical examination and Duplex scanning were found satisfactory on 14th postoperative day and on 3rd and 6th month. The fistula was mature by 6 weeks to start dialysis. The patient is having regular hemodialysis with this brachio-axilary fistula with translocation graft.

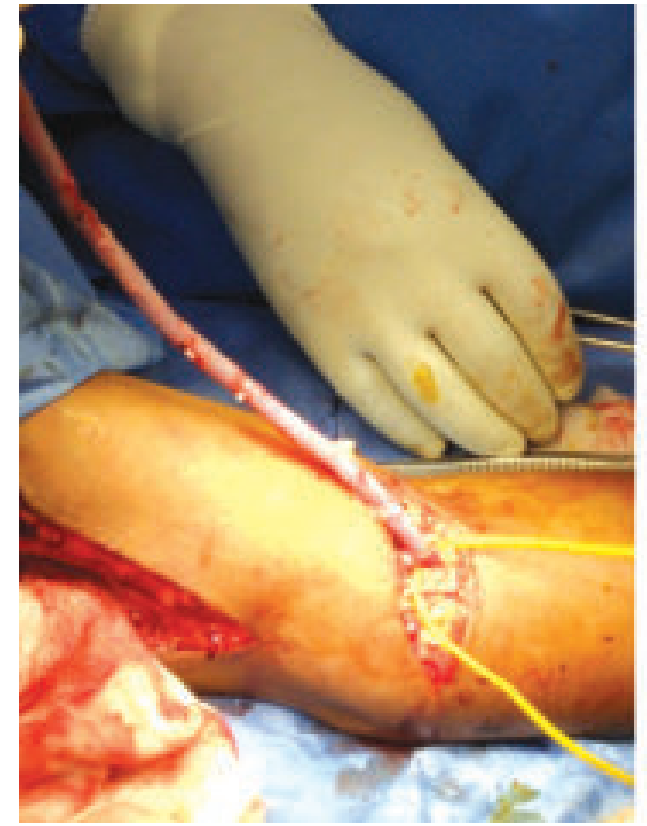

A

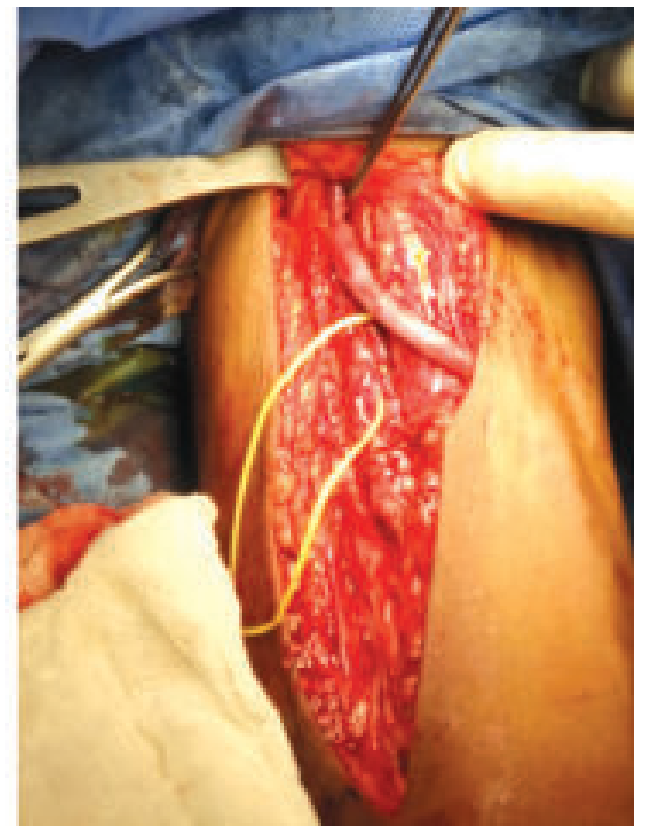

B

Fig.-1: A. Brachiobasilic Translocation fistula: Proximal Anastomosis. B. Distal Anastomosis. 


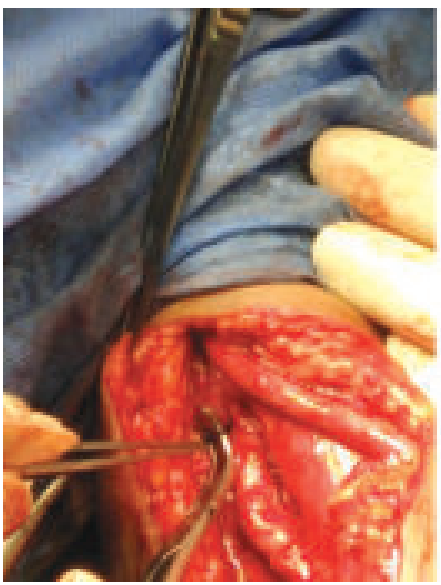

A

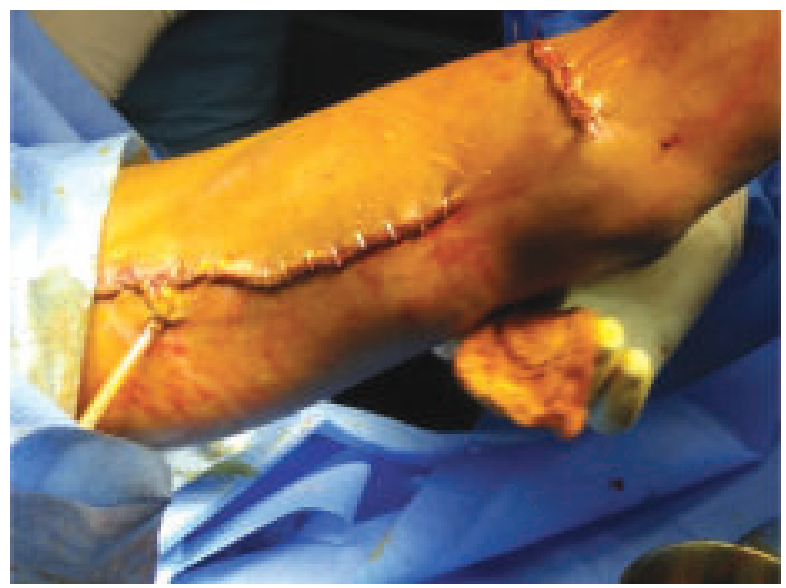

B

Fig.-2: A. Banding of distal Axillary vein to prevent venous hypertension of left upper limb. B. After completion of surgery.

\section{Discussion:}

It is quite evident that, whenever possible, native fistula should be preferred over prosthetic grafts. Distal upper arm fistulas involving radial artery is the first choice. If this or Bracial-Basilic fistulas are not possible then of upper arm cephalic and basilic veins with transpositions and great sapheneous vein with translocation wherever required can enhance autogenous fistula options to a large extent. Upper arm grafts should be used when no autogenous fistula is possible. Lower limb and body wall fistula sites are to be considered at the end, when all options in both upper limbs are exhausted. Autogenous arteriovenous fistulas (AVF) are the preferred mode of vascular access for maintenance hemodialysis (HD) in patients with end-stage renal disease (ESRD) because of their good long-term patency and low complication rate.

As the life expectancy of patients on long-term HD has improved with better healthcare facilities, most of them now stay on maintenance HD for much longer periods of time. Their vascular access also needs maintenance and management of various related complications. There is a large group of patients in whom Brescia-Cimino AVF has either failed in both upper limbs or is not feasible because of unsuitable veins. The best possible approach in the management of these patients to get a good long-term vascular access has been a matter of debate and discussion over the years.

The saphenous vein may be transposed either straight or in a loop fashion to make AVF. There are conflicting results in the literature about the outcome of sapheno-femoraltransposed fistulae. Lynggaard reported very poor patency rates and unacceptably high complications in their series ${ }^{3}$. Pierre-Paul et al. published their results that straight transposition of saphenous vein has better outcomes compared with loop configuration ${ }^{4}$. The mean primary patency was 7 months, primary-assisted patency was 15 months and secondary patency was 16 months. The fistula was functional for hemodialysis in $71.4 \%$. But compared to its native location Great Saphenous venous grafts may be much more patent when used as a free translocation graft in creating an upper limb AV fistula.

Prosthetic grafts have inferior primary and secondary patency rates and higher incidence of some complications such as infections and thrombosis compared with autogenous fistulae ${ }^{5,6,7}$. As a result, the last decade has seen a gradual and intentional shift toward increasing the use of autogenous $\mathrm{AVF}^{8,9}$. Autologous great saphenous grafts may be a very useful tool for the surgeons in creating upper limb AV fistulas in difficult situations.

\section{References:}

1. Srivastava A and Sharma S. Hemodialysis vascular access options after failed Brescia-Cimino arteriovenous fistula. Indian J Urol. 2011 Apr-Jun; 27(2): 163-168.

2. Santoro D, Benedetto F, Mondello P, Pipitò N, Barillà D, Spinelli F, Ricciardi CA, Cernaro V, Buemi M. Vascular access for hemodialysis: current perspectives. Int J Nephrol Renovasc Dis. 2014; 7: 281-94.

3. Lynggaard F, Nordling J, Iversen Hansen R. Clinical experience with the saphena loop arteriovenous fistula on the thigh. Int Urol Nephrol. 1981;13:287-90.

4. Pierre-Paul D, Williams S, Lee T, Gahtan V. Saphenous vein loop to femoral artery arteriovenous fistula: A practical alternative. Ann Vasc Surg. 2004;18:223-7. 
5. Coburn MC, Carney WI., Jr Comparison of basilic vein and polytetrafluoroethylene for brachial arteriovenous fistula. J Vasc Surg. 1994;20:896-902.

6. Murad MH, Elamin MB, Sidawy AN, Malaga G, Rizvi AZ, Flynn DN, et al. Autogenous versus prosthetic vascular access for hemodialysis: A systematic review and meta-analysis. J Vasc Surg. 2008;48:34S-7S.

7. Gibson KD, Gillen DL, Caps MT, Kohler TR, Sherrard DJ, Stehman-Breen CO. Vascular access survival and incidence of revisions: A comparison of prosthetic grafts, simple autogenous fistulas, and venous transposition fistulas from the United States Renal Data System Dialysis Morbidity and Mortality Study. J Vasc Surg. 2001;34:694-700.

8. Strott KL, Rodgers DJ, Karp SK, Woodruff SD, Wright LD. Increasing the use of arteriovenous fistulas (AVF): A network QI project. Nephrol News Issues. 2004;18:49-53.

9. National Kidney Foundation's KDOQI 2006 Vascular access Guidelines. Am J Kidney Dis. 2006;48: S177-322. 